\title{
Guest editorial: recent advances in the genetic basis of childhood hemato-oncological diseases
}

\author{
Shuki Mizutani
}

Received: 8 November 2012/Revised: 4 December 2012/ Accepted: 4 December 2012/Published online: 10 January 2013

(C) The Japanese Society of Hematology 2013

Recent advances in molecular biology brought on by rapid progress in the development of techniques for genetic and epigenetic analyses have ushered in a new era for discoveries relating to the genetic basis of childhood hematooncological diseases. A growing body of evidence indicates that inherited genetic traits may contribute substantially to the development of these diseases, including childhood leukemia. These traits can range from predisposing highly penetrant mutations to low-penetrant genetic polymorphisms. In this review series, four investigators and their colleagues were invited to introduce and discuss recent progress in gaining a deeper understanding of the genetic basis of childhood leukemia/cancer, as well as bone marrow failure syndromes.

Leukemia is the most common cancer in children and is characterized by the dysregulated proliferation of clonally expanded immature hematopoietic stem or progenitor cells that have undergone transformation through alterations within oncogenes, tumor suppressor genes, or genes involved in epigenetic control of cell proliferation. Similar to most cancers, the majority of childhood leukemia is thought to occur sporadically, with an etiology that likely involves exposure to carcinogenic environmental factors. Compared with adults, children typically have less cumulative exposure to environmental risk factors; however, cancer, particularly leukemia, nonetheless develops in children, suggesting that a significant genetic contribution underlies the development of childhood cancer.

S. Mizutani $(\bowtie)$

Department of Pediatrics and Developmental Biology,

Tokyo Medical and Dental University, 1-5-45 Yushima,

Bunkyo-ku, Tokyo 113-8519, Japan

e-mail:skkmiz@gmail.com
As one representative example of the involvement of low penetrance susceptibility alleles, Dr. Kevin Urayama and colleagues describe recent progress in identifying genetic risk factors for childhood acute lymphoblastic leukemia (ALL), the most common subtype of childhood leukemia. Dr. Urayama is a member of the Childhood Leukemia International Consortium (CLIC, https://ccls.berkeley.edu/ clic), a cooperative effort involving 22 epidemiological studies of childhood leukemia representing 12 countries, which was initiated in 2006 through the leadership of Professor Patricia Buffer of the University of California, Berkeley. In this low penetrance setting, genetic risk factors are thought to interact with environmental factors to modulate disease risk, making the continued study of both genetic and environmental risk factors for this disease essential.

A highly penetrant genetic influence is exemplified in the case of RAS/MAPK syndromes, introduced by Dr. Yoko Aoki. Noonan syndrome (NS), an autosomal-dominant disease characterized by distinctive facial features, webbed neck, cardiac anomalies, short stature, and cryptorchidism is a one such syndrome, in which germline mutations in genes encoding proteins of the RAS/mitogen-activated protein kinase (MAPK) pathway were discovered in elegant studies by Aoki et al. In her review, she describes the known genetic mutations, clinical manifestations, associations with malignant tumors, and possible therapeutic approaches for the disorders that comprise the RAS/MAPK syndrome.

The syndrome referred to as XCIND, first proposed by Professor Richard Gatti, is characterized by distinct hypersensitivity to ionizing (X-ray) irradiation. Patients often show concomitant cancer susceptibility, immunodeficiency, neurological abnormalities, and DNA double-strand break repair dysfunction, for which the syndrome is named. Such disorders are typically inherited in an autosomal recessive 
manner. My group discusses ataxia telangiectasia (A-T) as a representative XCIND disease.

Inherited bone marrow failure syndromes (IBMFs) are a heterogeneous group of genetic disorders characterized by bone marrow failure, congenital anomalies, and an increased risk of malignant disease. Representative diseases with trilineage involvement are Fanconi anemia and dyskeratosis congenita, while Diamond-Blackfan anemia manifests as a single lineage cytopenia. Our increased understanding of these diseases has come from the identification of genetic lesions involved in underlying molecular pathways, a field of study that continues to be driven by rapid advances in genomic technologies. Recent progress in genetic and molecular biological studies and treatment modalities of these diseases is discussed by Dr. Seiji Kojima and colleagues.

Research into the genetic basis, whether low-penetrant modified by environment factors or highly penetrant pathogenic mutations, of childhood hemato-oncological diseases, including those described in this review series, continues to aid in our understanding of the molecular mechanisms involved in disease pathogenesis, and brings us closer in our pursuit to develop new modalities for treatment and/or prevention. 